\title{
Infecções congênitas em um hospital público de referência em Macaé, Rio de Janeiro, no biênio 2016-2017
}

\section{Congenital infections in a public hospital of reference in Macaé, Rio de Janeiro, in the biennium 2016-2017}

\begin{abstract}
Kelly Mariana Pimentel Queiroz ${ }^{1}$, Hugo Demesio Maia Torquato Paredes², Ana Carolina Souza da Costa ${ }^{3}$, Mariana Oliveira do Couto Silva ${ }^{4}$,

Fernanda Valentim da Costa ${ }^{5}$, Luciana Aguiar Velasco Lima ${ }^{6}$, Cleber Nascimento do Carmo ${ }^{7}$, Jane de Carlos Santana Capelli ${ }^{8}$, Vivian de Oliveira Sousa Corrêa ${ }^{9}$
\end{abstract}

1. ORCID: https://orcid.org/0000-0001-8769-9835. Enfermeira. Curso de Enfermagem da Universidade Federal do Rio de Janeiro/Campus UFRJ-Macaé Professor Aloisio Teixeira, Rio de Janeiro, Brasil.

E-mail: kelly.pimentel@hotmail.com

2. ORCID: https://orcid.org/0000-0001-9723-9867. Enfermeiro. Mestre em Enfermagem. Curso de Enfermagem da Universidade Federal do Rio de Janeiro/Campus UFRJ-Macaé Professor Aloisio Teixeira, Rio de Janeiro, Brasil.

E-mail: hugomaia2007@hotmail.com

3. ORCID: https://orcid.org/0000-0002-9404-4201. Bolsista de Iniciação Científica - PIBIC/UFRJ. Graduanda do Curso de Medicina da Universidade Federal do Rio de Janeiro/Campus UFRJ-Macaé Professor Aloisio Teixeira, Rio de Janeiro, Brasil.

E-mail: anasouzaufri@gmail.com

4. ORCID. https://orcid.org/0000-0002-5458-2910. Bolsista de Iniciação Científica - PIBIC/CNPq. Graduanda do Curso de Medicina da Universidade Federal do Rio de Janeiro/Campus UFRJ-Macaé Professor Aloisio Teixeira, Rio de Janeiro, Brasil.

E-mail: mariana.couto2015@outlook.com

5. ORCID. https://orcid.org/0000-0001-7979-0487. Graduanda do Curso de Medicina da Universidade Federal do Rio de Janeiro/Campus UFRJ-Macaé Professor Aloisio Teixeira, Rio de Janeiro, Brasil.

E-mail: nandavalentim.fvc@gmail.com

6. ORCID: https://orcid.org/0000-0002-6349-8091. Fonoaudióloga. Especialista em Disfagia e Desenvolvimento da Linguagem. Fundação Municipal Hospitalar de Macaé/Secretaria Municipal Adjunta de Alta e Média Complexidade de Macaé, Macaé Rio de Janeiro, Brasil.

E-mail: luka.velasco@globo.com

7. ORCID: https://orcid.org/0000-0003-4165-2198. Estatístico. Doutor em Ciências. Escola Nacional de Saúde Pública Sérgio Arouca/Fiocruz, Rio de Janeiro, Brasil.

E-mail. cleber.carmo@ensp.fiocruz.br 
8. ORCID: https://orcid.org/0000-0002-8009-3715. Nutricionista. Doutora em Ciências. Universidade Federal do Rio de Janeiro/Campus UFRJ-Macaé Professor Aloisio Teixeira, Rio de Janeiro, Brasil.

E-mail: jcscapelli@gmail.com

9. ORCID: https://orcid.org/0000-0002-3294-3703. Fisioterapeuta. Doutora em Ciências Morfológicas. Universidade Federal do Rio de Janeiro/Campus UFRJMacaé Professor Aloisio Teixeira, Rio de Janeiro, Brasil.

E-mail: profa.viviansousa@gmail.com

CONTATO: Autor correspondente: Jane de Carlos Santana Capelli | Endereço: Curso de Nutrição. Universidade Federal do Rio de Janeiro/Campus UFRJ-Macaé Professor Aloisio Teixeira. Rua Aloísio da Silva Gomes, 50 - Granja dos Cavaleiros. Macaé - Rio de Janeiro, Brasil. CEP: 27930-560 Telefone: (21) 974406176 E-mail: jcscapelli@gmail.com

\section{RESUMO}

As infecções congênitas estão relacionadas à mortalidade fetal e neonatal e fazem parte dos Indicadores de Risco para a Deficiência Auditiva previstos na Triagem Auditiva Neonatal. O estudo objetiva descrever a proporção de infecções congênitas no Programa de Triagem Auditiva Neonatal do Hospital Público Municipal de Macaé, Rio de Janeiro. Realizou-se um estudo retrospectivo, descritivo, de base secundária, a partir dos registros de neonatos obtidos no setor de Fonoaudiologia, entre 2016 e 2017. No biênio, detectaram-se 114 neonatos com infecções congênitas, 36,0\% ( $n=41)$ em 2016, e 64,0\% ( $n=73$ ) em 2017. Destes, 46,3\% e 46,6\% apresentavam sífilis congênita; $29,3 \%$ e $24,7 \%$ o vírus da imunodeficiência humana; $17,1 \%$ e $15,1 \%$ toxoplasmose, em 2016 e 2017, respectivamente. O aumento de infecção por citomegalovírus no período passou de 2016 (2,4\%) para 2017 $(8,2 \%)$. Conclui-se que as infecções congênitas de maior proporção foram a sífilis congênita, o vírus da imunodeficiência humana e a toxoplasmose.

DESCRITORES: Indicador de Risco. Perda Auditiva. Política Pública. Saúde Materno-Infantil. Triagem Neonatal.

\section{ABSTRACT}

Congenital infections are related to fetal and neonatal mortality and are part of the Risk Indicators for Hearing Impairment provided for in the Neonatal Hearing Screening. This study aims to describe the proportion of congenital infections in the Neonatal Hearing Screening Program of the Municipal Public 
Hospital Public in Macaé, Rio de Janeiro. A retrospective, descriptive, secondary-based study was carried out from the records of newborns obtained in the Speech Therapy sector, between 2016 and 2017. In the biennium, 114 cases of congenital infections in neonates were detected, $36.0 \%(n=41)$ in 2016, and $64.0 \%(n=73)$ in 2017 . Of these, $46.3 \%$ and $46.6 \%$ had congenital syphilis; $29.3 \%$ and $24.7 \%$ the human immunodeficiency virus; $17.1 \%$ and $15.1 \%$ toxoplasmosis, in 2016 and 2017, respectively. The increase in cytomegalovirus infection in the period went from 2016 (2.4\%) to 2017 (8.2\%). It is concluded that the highest proportion of congenital infections were syphilis, human immunodeficiency virus and toxoplasmosis.

DESCRIPTORS: Risk Index. Hearing Loss. Public Policy. Maternal and Child Health. Neonatal Screening. 


\section{INTRODUÇÃO}

s infecções congênitas são aquelas transmitidas ao feto ainda na gestação ou

durante o parto, e consideradas importantes causas de mortalidade fetal e neonatal e contribuem expressivamente para a morbidade infantil ${ }^{1}$, como sequelas e prejuízos na audição ${ }^{2,3}$.

No grupo de infecções congênitas que apresentam agentes etiológicos diferentes, mas com características clínicas similares ${ }^{4}$ encontram-se a toxoplasmose, a rubéola congênita, o citomegalovírus, a herpes, a sífilis congênita e o vírus da imunodeficiência humana (HIV), sendo sumarizadas pela sigla TORSCH-A ${ }^{3}$, sendo investigados em gestantes na assistência pré-natal ${ }^{4}$, e em neonatos na Triagem Auditiva Neonatal (TAN) ${ }^{5}$. A TAN visa detectar precocemente a deficiência auditiva nos recém-nascidos (RN) e lactentes, devendo fazer parte da Rede de Cuidados à Pessoa com Deficiência e às ações de acompanhamento do grupo materno-infantili5.

Estudos têm revelado a associação entre determinados tipos de infecções congênitas e a deficiência auditiva ${ }^{6,7}$ como o citomegalovírus, a rubéola, a toxoplasmose e a sífilis, sendo incluídas no grupo de indicadores de risco para deficiência auditiva - IRDA, que fazem parte do conjunto de ações da TAN².

A deficiência auditiva é uma das deficiências sensoriais de maior prevalência no mundo, e a infecção congênita por citomegalovírus (CMV) se constitui na principal causa de perda auditiva neurossensorial em crianças ${ }^{9}$.

No Brasil, o estudo comparando a ocorrência dos IRDA ao longo do período de janeiro de 2012 a dezembro de 2016, em um centro de saúde auditiva de média complexidade do estado do Rio Grande do Sul, em uma amostra 2.333 neonatos apresentando pelo menos um indicador de risco para a deficiência auditiva, detectou a tendência significativa para 0 aumento da toxoplasmose congênita, da sífilis congênita, do HIV congênito, além da hereditariedade e do nascimento pré-termo. Os autores observaram que a infecção congênita de maior incidência foi a sífilis ${ }^{10}$.

No município de Macaé, um estudo observacional descritivo retrospectivo, analisando o perfil epidemiológico dos casos de sífilis notificados na cidade de Macaé, Rio de Janeiro, entre 2013 e 2016, obtidos por meio de registros fornecidos pela 
Vigilância Epidemiológica/Sistema de Informação de Agravos de Notificação (Sinan) do município, encontraram 255 casos de sífilis em gestantes, sendo 133 em 2016; destes, 35 casos eram sífilis congênita ${ }^{11}$.

Considerando a relevância do tema no campo das políticas públicas voltadas a saúde da mulher e da criança e os poucos estudos no Brasil sobre o tema, o presente estudo objetiva descrever a proporção de infecções congênitas no Programa de Triagem Auditiva Neonatal do Hospital Público Municipal de Macaé, Rio de Janeiro.

\section{MÉTODO}

Realizou-se um estudo descritivo, retrospectivo, quantitativo, de base secundária, abrangendo 5.385 RN que foram submetidos ao Programa de Triagem Auditiva Neonatal (PTAN) da maternidade do Hospital Público Municipal Dr. Fernando Pereira da Silva (HPM), nos anos 2016 ( $n=2.479$ nascidos vivos) e 2017 ( $n=2.806$ nascidos vivos), segundo dados fornecidos pelo setor de vigilância epidemiológica do hospital. O HPM está localizado em Macaé, estado do Rio de Janeiro, município da região do Norte Fluminense, com estimativa populacional de 206.728 habitantes $^{12}$.

Todos os RN vivos entre janeiro e dezembro de 2016 e 2017, que passaram pela PTAN da maternidade do HPM e que apresentaram pelo menos um indicador de risco para deficiência auditiva foram incluídos no estudo. Foram excluídos os $\mathrm{RN}$ que não eram residentes do município de Macaé e não apresentaram IRDA.

As variáveis de estudo foram: sexo do neonato; local de realização do exame (Unidade de Terapia Intensiva Neonatal - UTIN; Alojamento Conjunto - AC); idade gestacional (<37 semanas, pré-termo; 37 a 41, a termo; >=42 semanas, pós-termo); teste de emissão otoacústica evocada por estímulo transiente - EOAT (passou ou falhou), associado a presença ou ausência do reflexo cócleo-palpebral - RCP, conforme rege o protocolo de triagem do hospital; e as infecções congênitas como o IRDA $^{2}$.

A coleta de dados ocorreu no período entre agosto de 2016 e fevereiro de 2018, por dois auxiliares de pesquisa, em dois turnos da semana, que anotavam as informações contidas em um livro de registro dos RN que passavam pela PTAN, no 
setor de Fonoaudiologia do HPM, utilizando-se um formulário estruturado para cada $\mathrm{RN}$, elaborado e testado previamente para o estudo.

As variáveis foram sumarizadas por meio de estatísticas descritivas, frequências simples e relativas. Verificou-se a significância das diferenças encontradas nas respostas por grupos e categorias pelo teste estatístico quiquadrado. As análises estatísticas foram conduzidas utilizando-se o software Statistical Package for the Social Sciences, versão 20.0 (SPSS, Chicago, IL) e o nível de significância de $5 \%$ considerado.

O projeto de pesquisa foi aprovado pelo Comitê de Ética em Pesquisa da Faculdade de Medicina de Campos/Fundação Benedito Pereira Nunes, sob parecer ํo. 660.225 .

\section{RESULTADOS}

No biênio, 5.285 neonatos foram submetidos ao PTAN, dos quais $77,2 \%$ ( $n=1.914$ ) foram avaliados em 2016 ( $n$ total=2.479) e 74,5\% ( $n=2.090)$ em 2017 ( $n$ total=2.806). Nesse período, 3,8\% ( $n=201)$ dos neonatos apresentaram pelo menos um IRDA. Analisando por ano, em 2016, 4,5\% ( $n=86)$ dos neonatos apresentaram pelo menos um IRDA e em 2017, 5,5\% ( $n=115)$ (dados não apresentados em tabela).

Na Tabela 1, no ano de 2016, 57,0\% dos neonatos eram do sexo masculino, $58,1 \%$ realizaram o EOAT na UTIN, 38,4\% nasceram pré-termo, 33,7\% falharam no EOAT e $91,9 \%$ passaram no RCP. Em 2017, 50,4\% eram do sexo feminino, 87,0\% realizaram o EOAT na UTIN, 29,6\% nasceram pré-termo, 21,7\% falharam no EOAT e 95,7\% passaram no RCP. Ao comparar a realização do EOAT na UTIN nos anos 2016 e 2017, detectou-se a diferença estatisticamente significativa ( $p$ valor $<0,001)$ (Tabela 1). 
Tabela 1. Características dos neonatos submetidos ao Programa de Triagem Auditiva Neonatal (PTAN) e apresentaram pelo menos um Indicador de Risco para Deficiência Auditiva, no Hospital Público de Macaé, 2016-2017.

\begin{tabular}{lccc}
\hline Características & $\begin{array}{c}2016(\mathbf{n}=86) \\
\mathbf{n}(\%)\end{array}$ & $\begin{array}{c}\mathbf{2 0 1 7}(\mathbf{n}=\mathbf{1 1 5}) \\
\mathbf{n}(\%)\end{array}$ & p valor \\
\hline Sexo & & & \\
Feminino & $37(43,0)$ & $58(50,4)$ & 0,184 \\
Masculino & $49(57,0)$ & $57(49,6)$ & \\
Local de realização do exame & & & \\
UTIN ${ }^{1}$ & $50(58,1)$ & $100(87,0)$ & $<0,001$ \\
Alojamento Conjunto & $36(41,9)$ & $15(13,0)$ & \\
Idade gestacional do neonato & & & \\
Pré-termo & $33(38,4)$ & $34(29,6)$ & \\
A termo & $52(60,5)$ & $79(68,7)$ & 0,414 \\
Pós-termo & $1(1,1)$ & $2(1,7)$ & \\
EOAT & & & \\
Passou & & & \\
Falhou & $57(66,3)$ & $90(78,3)$ & 0,176 \\
Reflexo cócleo-palpebral & $29(33,7)$ & $25(21,7)$ & \\
Passou & & & \\
Falhou & $79(91,9)$ & $110(95,7)$ & 0,556 \\
\hline
\end{tabular}

Fonte: Elaborada pelos autores (2021).

Legenda: ${ }^{1}$ Unidade de Terapia Intensiva Neonatal, ${ }^{2}$ Teste de Emissão Otoacústica Evocada por Estímulo Transiente.

No ano de 2016, dos 86 neonatos que apresentaram pelo menos um IRDA, 47,7\% ( $n=41)$ tinham infecção congênita. Em 2017, dos 115 neonatos com pelo menos um IRDA, 63,5\% ( $n=73$ ) tinham infecção congênita (dados não apresentados em tabela).

A Figura 1 apresenta as proporções das infecções congênitas detectadas no PTAN. Dentre os casos de infecções congênitas em 2016 e 2017, 46,3\% e 46,6\% eram sífilis congênita; $29,3 \%$ e $24,7 \%$ eram do vírus da imunodeficiência humana (HIV); $17,1 \%$ e $15,1 \%$ eram toxoplasmose, respectivamente. Cabe ressaltar o aumento de infecção por citomegalovírus de 2016 (2,4\%) para 2017 (8,2\%) (Figura 1). 
Figura 1. Distribuição percentual das infecções congênitas no Programa de Triagem Auditiva Neonatal, do Hospital Público de Macaé, 2016-2017.

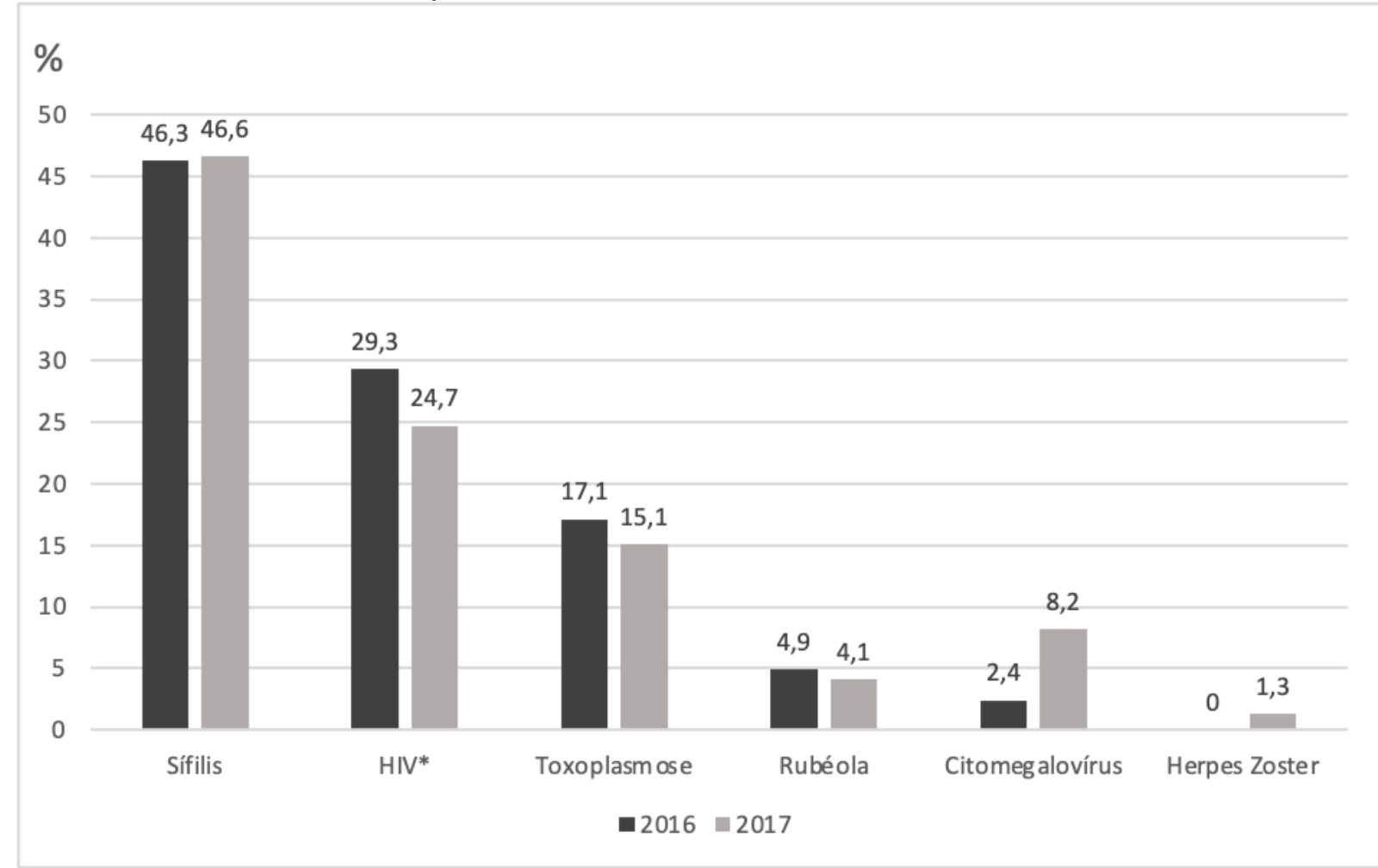

Fonte: Elaborada pelos autores (2021).

Legenda:*HIV: Vírus da Imunodeficiência Humana.

$2016(n=41)$.

$2017(n=73)$.

\section{DISCUSSÃO}

Neste estudo, detectou-se que as infecções congênitas de maior proporção no biênio foram a sífilis, o HIV e a toxoplasmose. Além disso, a proporção da infecção por citomegalovírus quadruplicou de 2016 para 2017.

A Organização Mundial da Saúde (OMS) estima que 60,0\% da perda auditiva infantil seja devido a causas evitáveis, dentre elas as infecções congênitas ${ }^{13}$. Estudos mostram que os agentes infecciosos de maior prevalência associados a perda auditiva são: citomegalovírus, vírus da rubéola, Toxoplasma gondii, vírus herpes ${ }^{14} \mathrm{e} \mathrm{HIV}^{15}$. No entanto, a sífilis foi a principal causa das infecções congênitas neste estudo.

As infecções congênitas são transmitidas frequentemente por via hematogênica transplacentária, depois a infecção materna, ou, raramente, por via ascendente (por meio do colo do útero), no decorrer da gestação. A maioria das infecções congênitas é assintomática, tanto para a mãe quanto para o feto, como a 
rubéola, o citomegalovírus, a toxoplasmose e a herpes simples. No entanto, podem gerar danos ao feto ao longo da gestação como, por exemplo, o retardo do crescimento intrauterino, o aborto espontâneo, a malformação e a surdez ${ }^{16}$.

Estudo avaliando 140 prontuários de neonatos internados em UTIN por mais de cinco dias, detectou que 2,1\% apresentavam infecções congênitas ${ }^{17}$. A pesquisa realizada em uma unidade neonatal de um hospital municipal da zona norte de São Paulo, detectou 1.805 neonatos foram submetidas a Triagem Auditiva Neonatal, e, deste total, apenas $14,1 \%(n=221)$ apresentaram um ou mais IRDA, além da prevalência de $2,7 \%$ de infecções congênitas ${ }^{18}$. Esses dados divergem deste estudo, que apresentou maior proporção tanto para presença de um ou mais IRDA como infecções congênitas nos RN.

A sífilis ainda é considerada problema de saúde pública no mundo, detectandose 1,36 milhões de gestantes infectadas anualmente, das quais $80,0 \%$ fizeram o prénatal ${ }^{19}$. No Brasil, a sífilis congênita, é considerada um evento-sentinela de monitoramento na Atenção Primária em Saúde devido a sua facilidade de prevenção, cujo aparecimento indica possíveis falhas na operacionalização da Rede de Atenção Básica ${ }^{16}$.

A transmissão da sífilis ocorre principalmente pelo contato sexual, porém devese considerar outras formas de transmissão como, por exemplo, o contato do lactente por meio da amamentação. A lactante que possui sífilis primária ou secundária pode contaminar a criança por meio de lesões que possam apresentar na mucosa das mamas $^{20}$.

Os casos de sífilis congênita estão aumentando, apresentando uma incidência de 1,3/1000 nascidos vivos nas Américas e, no Brasil, 6,5/100021. Entre 2005 e 2016, o Sistema de Informações de Agravos de Notificação (Sinan) notificou 169.546 casos de sífilis em gestantes brasileiras ${ }^{22}$.

O estudo visando caracterizar o perfil epidemiológico dos recém-nascidos de risco com infecção congênita atendidos na Unidade de Referência Materno Infantil e Adolescente/URE-MIA, no município de Belém/Pará, no período entre 2008 e 2013, em $186 \mathrm{RN}$, detectou $147(79,0 \%)$ casos de sífilis, do total dos neonatos, seguida por toxoplasmose e citomegalovirose ${ }^{16}$. Neste estudo, a proporção de $\mathrm{RN}$ com sífilis foi inferior quando comparada a encontrada ao da pesquisa realizada em Belém. No 
entanto, em ambos os estudos, os dados são preocupantes devido as consequências negativas ao feto como a prematuridade e o baixo peso ao nascer ${ }^{20}$.

No município de Macaé/Rio de Janeiro, o estudo observacional descritivo retrospectivo, analisando o perfil epidemiológico dos casos de sífilis notificados, no período entre 2013 e 2016, obtidos por meio de registros fornecidos pela Sinan do município, detectou que, de 1.047 casos, 86 foram notificados no ano de 2013; 177 em 2014; 228 em 2015 e 556 em 2016, revelando uma tendência ascendente da doença. As autoras encontraram 690 casos notificados de sífilis adquirida: 67, em 2013; 110 em 2014; 125 em 2015; e 388 em 2016. Em gestantes, observaram 255 casos de sífilis, sendo 15 em 2013; 38 em 2014; 69 em 2015; e 133 em 2016. Observaram também 102 casos de sífilis congênita: 4 em 2013; 29 em 2014; 34 em 2015; e 35 em 2016, dentre eles um caso de óbito ${ }^{11}$.

Os dados supracitados revelam um aumento expressivo dos casos de sífilis no município de Macaé, corroborando a elevada proporção de sífilis congênita nesse estudo. Além disso, os achados são preocupantes, pois, como a sífilis pode ser assintomática, a detecção tardia no feto possivelmente acarretará prejuízos ao feto como a surdez, o retardo mental, o arco palatino elevado, a hidrocefalia, dentre outros $^{20}$.

A toxoplasmose, nesse estudo, foi a terceira infecção congênita mais frequente, apesar de seu decréscimo no biênio estudado. No Brasil, a prevalência de toxoplasmose em gestantes é de 31,1\%, em Caxias do sul é de $91,6 \%$, e de $91,6 \%$, em Mato Grosso do Sul, em Sergipe, é de 69,3\% e em sua capital, 77,8\%²2. Em estudo realizado em um centro de saúde do Rio Grande do Sul, houve um cenário de redução semelhante $^{8}$. No entanto, tal conjuntura não exclui a indicação do rastreamento de gestantes visando melhorar o tratamento materno e prevenir a transmissão vertical ${ }^{1}$.

O estudo realizado no Programa de Triagem Auditiva Neonatal de Minas Gerais, com 106 crianças diagnosticadas com toxoplasmose congênita, revelou que $43,4 \%$ apresentavam alterações audiológicas ${ }^{23}$. Segundo os autores, a avaliação diagnóstica, e, consequente detecção precoce na gestação, permite a intervenção na toxoplasmose rapidamente, e equivale a redução de um dos indicadores de perda auditiva de grande relevância ${ }^{24}$. 
Quanto ao citomegalovírus, nossa pesquisa revelou um aumento expressivo entre 2016 e 2017. No mundo, a infecção congênita por citomegalovírus afeta entre $0,7 \%$ e $6,0 \%$ dos neonatos ${ }^{25}$, estando abaixo daquele encontrado em nosso estudo no ano de 2017.

No Brasil, estudo realizado no serviço de otorrinolaringologia e cirurgia cérvicofacial do Hospital Garcia de Orta entre o ano de 2007 a 2015, revelou que 12 crianças apresentaram potencial para infecção congênita por citomegalovírus, e, destas, uma apresentou surdez sensorioneural ${ }^{26}$, ressaltando-se, portanto, a importância da detecção precoce e do acompanhamento regular a crianças com esse diagnóstico ${ }^{27}$.

Cabe ressaltar que parte dos neonatos submetidos a PTAN, nesse estudo, nasceram pré-termo [38,4\% ( $n=33)$, em 2016; e 29,6\%(n=34), em 2017, Tabela 1)] estando mais susceptíveis a apresentarem perda auditiva. Em 2012, dados da OMS revelaram o nascimento anual de aproximadamente 15 milhões de crianças pré-termo em todo o mundo, ficando o Brasil em décimo lugar em maior número absoluto ${ }^{28}$.

O presente estudo possui algumas limitações. Uma delas foi a utilização de dados secundários, não sendo possível a confiabilidade total na qualidade deles, devido aos erros de preenchimento. Contudo, as fonoaudiólogas que anotam os dados obtidos na PTAN no livro de registro do setor de Fonoaudiologia, do HPM, buscam estar atentas quanto ao seu preenchimento, segundo a coordenadora do setor. Além disso, o PTAN não atendeu cerca de 1/4 dos neonatos, o que impossibilita a generalização dos resultados para outras populações. No entanto, os resultados obtidos sinalizam para a necessidade de um olhar mais atento para a RAS no município e região, uma vez que é um lócus privilegiado para o cuidado centrado na pessoa.

Logo, é preciso fortalecer as ações de mapeamento e monitoramento das demandas e necessidades de saúde da população, em especial das mulheres em idade fértil, e não só na assistência pré-natal, adotando medidas de prevenção de doenças e promoção da saúde para reduzir o risco ao qual a população está exposta, tais como: a imunização de crianças contra doenças infantis, como sarampo, meningite, rubéola e caxumba; e de adolescentes e mulheres em idade reprodutiva contra a rubéola antes da gravidez; prevenção, diagnóstico precoce e tratamento de 
HIV, sífilis e outras infecções sexualmente transmissíveis em mulheres em idade fértil, gestantes, bem como a população em geral ${ }^{29}$.

Os investimentos na assistência pré-natal, promoção da saúde e prevenção de doenças no campo da saúde materno-infantil, em diferentes países do mundo, determinaram a redução da incidência das infecções congênitas nesse grupo populacional $^{30}$. No entanto, no Brasil, ainda há escassez de estudos voltados epidemiologia das infecções congênitas nos municípios e regiões que possam conhecer a sua magnitude, distribuição e fatores relacionados a morbimortalidade fetal e neonatal.

\section{CONCLUSÃo}

No biênio, as infecções congênitas mais frequentes no Programa de Triagem Auditiva Neonatal do HPM foram a sífilis, o HIV e a toxoplasmose. Além disso, houve maior crescimento da infecção por citomegalovírus de 2016 para 2017.

\section{REFERÊNCIAS}

1. Miranda MMS, Souza LMG, Aguiar RALP, Corrêa Jr. MD, Maia MMM, Borges RS, Melo VH. Rastreamento das infecções perinatais na gravidez: realizar ou não? Femina. [Internet]. 2012 [acesso em 2021 ago 12]; 40(1):12-22. Disponível em: http://files.bvs.br/upload/S/0100-7254/2012/v40n1/a3075.pdf

2. Brasil. Ministério da Saúde. Secretaria de Atenção à Saúde. Departamento de Ações Programáticas Estratégicas. Diretrizes de Atenção da Triagem Auditiva Neonatal. Brasília, 2012. Disponível em: https://bvsms.saude.gov.br/bvs/publicacoes/diretrizes atencao triagem auditiva neonatal.pdf

3. Lopes MKD, Santos TMM. Comparação dos Indicadores de Risco para Surdez em Neonatos Encontrados nos Anos de 1995 e 2005. Arq. int. otorrinolaringol. 2011 [Internet]. [acesso em 2021 ago 12]; 15(1):35-40. Disponível em: https://www.scielo.br/j/aio/a/WrSbfgy9rbptdK4NG49sMJq/abstract/?lang=pt

4. Pereira DAP, Maia BP, Seto IIC, Bichara CNC. Infecção congênita em pacientes matriculados em programa de referência materno infantil. Rev. para. med. 2015 [Internet]. [acesso em 2021 ago 12]; 29(1):31-38. Disponível em: https://pesquisa.bvsalud.org/portal/resource/pt/lil-747241

5. Brasil. Ministério da Saúde. Secretaria de Atenção à Saúde. Departamento de Ações Programáticas e Estratégicas. Atenção à saúde do recém-nascido: guia 
para os profissionais de saúde/Ministério da Saúde, Secretaria de Atenção à Saúde, Departamento de Ações Programáticas e Estratégicas. - Brasília: Ministério da Saúde, 2011. 4 v.: il. - (Série A. Normas e Manuais Técnicas).

6. Nóbrega MD, Weckx LLM, Juliano Y. Study of the hearing loss in children and adolescents, comparing the periods of 1990-1994 and 1994-2000. Int. j. pediatr. otorhinolaryngol. 2005 [Internet]. [acesso em 2021 ago 12]; 69:829-38. doi: https://doi.org/10.1016/j.ijporl.2005.01.019

7. Arvin AM, Whitley RJ, Gutierrez KM. Herpes Simplex Virus Infections. In: Remington JS, Klein JO, Wilson CB, Baker CJ, editors. Infectious Diseases of the Fetus and Newborn Infant. 6th ed. Philadelphia: Elsevier Saunders; 2006.

8. Brasil. Ministério da Saúde. Secretaria de Atenção à Saúde. Departamento de Ações Programáticas Estratégicas. Diretrizes de Atenção da Triagem Auditiva Neonatal/Ministério da Saúde, Secretaria de Atenção à Saúde, Departamento de Ações Programáticas Estratégicas e Departamento de Atenção Especializada. Brasília: Ministério da Saúde, 2012. 32 p.: il.

9. Huang S-N, Zhou YP, Jiang X, Yang B, Cheng H, Luo M-H. Hearing Loss Caused by HCMV Infection through Regulating the Wnt and Notch Signaling Pathways. Viruses. 2021 [Internet]. [acesso em 2021 ago 12]; 13(4):623. doi: https://doi.org/10.3390/v13040623

10. Silva AA, Bento DV, Silva LNFB. Ocorrência dos indicadores de risco para a deficiência auditiva em um centro de saúde do Rio Grande do Sul. Audiol. commun. res. 2018 [Internet]. [acesso em 2021 ago 12]; 23:e1919-e1919. doi: https://doi.org/10.1590/2317-6431-2017-1919

11. Souza BSO, Rodrigues RM, Gomes RML. Análise epidemiológica de casos notificados de sífilis. Rev. soc. bras. clin. med. 2018 [Internet]. [acesso em 2021 ago 12]; 16(2):94-8. Disponível em: https://docs.bvsalud.org/biblioref/2018/09/913366/16294-98.pdf

12. Instituto Brasileiro de Geografia e Estatística. Macaé. Rio de Janeiro. Cidades [Internet]. Rio de Janeiro: Instituto Brasileiro de Geografia e Estatística; 2016 [internet]. [acesso em 2021 ago 12]. Disponível em: http://cidades.ibge.gov.br/xtras/perfil.php?codmun=330240.17

13. World Health Organization (WHO). Addressing the rising prevalence of hearing loss. February 2018. [Internet]; [cited 2021 ago 16]. Disponível em: https://apps.who.int/iris/bitstream/handle/10665/260336/9789241550260-eng.pdf

14. American Academy of Pediatrics, Joint Committee on Infant Hearing. Year 2007 position statement: principles and guidelines for early hearing detection and intervention programs. Pediatrics 2007 [internet]. [cited 2021 ago 12]; 120(4):898921. doi: https://doi.org/10.1542/peds.2007-2333

15. Assuiti LFC, Lanzoni GMM, Santos FC, Erdmann AL, Meirelles BHS. Perda auditiva em pessoas com HIV/AIDS e fatores relacionados: uma revisão 
integrativa. Braz. j. otorhinolaryngol. 2013 [Internet]. [acesso em 2021 ago 16]; 79(2): 248-255. doi: https://doi.org/10.5935/1808-8694.20130042

16. Rechia IC, Liberalesso KP, Angst OVM, Mahl FD, Garcia MV, Biaggio EPV. Unidade de Terapia Intensiva: resultados da Triagem Auditiva Neonatal. Braz. j. otorhinolaryngol. 2016 [Internet]. [acesso em 2021 ago 12]; 82(1): 76-81. doi: https://doi.org/10.1016/j.bjorl.2015.06.004

17. Onoda RM, Azevedo MF, Santos AMN. Triagem auditiva neonatal: ocorrência de falhas, perdas auditivas e indicadores de riscos. Braz. j. otorhinolaryngol. 2011 [Internet]. [acesso em 2021 ago 12]; 77(6):775-83. doi: https://doi.org/10.1590/S1808-86942011000600015

18. Soares LG, Zarpellon B, Soares LG, Baratieri T, Lentsck MH, Mazza VA. Gestational and congenital syphilis: maternal, neonatal characteristics and outcome of cases. Rev. bras. saúde mater. infant. 2017 [Internet]. [acesso em 2021 ago 11]; 17(4):781-789. doi: https://doi.org/10.1590/1806$\underline{93042017000400010}$

19. Sonda EC, Richter FF, Boschetti G, Casasola MP, Kromel CF, Machado CPH. Sífilis Congênita: uma revisão da literatura. Rev Epidemiol Control Infect. 2013 [Internet]. [acesso em 2021 nov 03]; 3(1):28-30. Disponível em: https://pdfs.semanticscholar.org/0e52/5d5386e2a0a4cab23d15a2274eec99c0d2 61.pdf

20. Pan American Health Organization. Elimination of mother-to-child transmission of HIV and syphilis in the Americas. Washington: PAHO; 2014. Disponível em: https://iris.paho.org/bitstream/handle/10665.2/18372/9789275118702 eng.pdf?s equence $=3 \&$ is Allowed $=y$

21. Brasil. Ministério da Saúde. Secretaria de Vigilância em Saúde. Departamento de DST, Aids e Hepatites Virais. Boletim Epidemiológico da Sífilis. Brasília. 2016 [internet]. [acesso em 2021 ago 11]; 35(47). Disponível em: http://www.aids.gov.br/pt-br/pub/2016/boletim-epidemiologico-de-sifilis-2016

22. Inagaki ADM, Cardoso NP, Lopes RJPL, Alves JAB, Mesquita JRF, Araújo KCGM, et al. Análise espacial da prevalência de toxoplasmose em gestantes de Aracaju, Sergipe, Brasil. Rev. bras. ginecol. obstet. 2014 [Internet]. [acesso em 2021 ago 12]; 36(12):535-540. doi: https://doi.org/10.1590/So100-720320140005086

23. Resende LM, Andrade GMQ, Azevedo MF, Perissinoto J, Vieira ABC. Congenital toxoplasmosis: Auditory and language outcomes in early diagnosed and treated children. Sci Med. 2010 [Internet]. [acesso em 2021 ago 12]; 20(1):13-19. Disponível

em: https://revistaseletronicas.pucrs.br/ojs/index.php/scientiamedica/article/download $\underline{15927 / 4875 /}$

24. Hampton MM. Congenital toxoplasmosis: a review. Neonatal Netw. 2015 [Internet]. [acesso em 2021 ago 12]; 34(5):274-8. doi: https://doi.org/10.1891/0730$\underline{0832.34 .5 .274}$ 
25. Tagarro A, Del Valle R, Dominguez-Rodríguez $S$, Baquero-Artigao $F$, NogueraJulian A, Vives-Onos I, et al. Growtth patterns in children with congenital cytomegalovirus infection. Pediatr infect. dis. J. 2019 [Internet]. [acesso em 2021 ago 12]; 39(12):1230-1235. doi: https://doi.org/10.1097//NF.0000000000002483

26. Alves FS, Caixa M, Rosa MH, Santos M, Antunes L. Perfil audiométrico das crianças com diagnóstico de infecção congénita por citomegalovírus - Dados de 9 anos de consulta de surdez infantil. Rev. Port. ORL. 2018 [Internet]. [acesso em 2021 ago 12]; 56(2):61-65. doi: https://doi.org/10.34631/sporl.681

27. Vernier LS, Castelli CTR, Rothermel SS, Paniz TC, Zanini C, Levandowski DC. Triagem auditiva neonatal em hospital da rede cegonha. Rev. bras. promoç. saúde. 2019 [Internet]. [acesso em 2021 ago 16]; 32:8965. doi: https://doi.org/10.5020/18061230.2019.8965

28. World Health Organization. March of Dimes; The Partnership for Maternal Newborn and Child Health; Save the Children; World Health Organization. Born too soon: the global action report on preterm birth. Geneva: WHO; 2012. Disponível em: http://apps.who.int/iris/bitstream/handle/10665/44864/9789241503433 eng.pdf?s equence $=1$

29. Pereira PKS, Martins AS, Vieira MRV, Azevedo MF. Programa de triagem auditiva neonatal: associação entre perda auditiva e fatores de risco. Pró-fono. 2007 [Internet]. [acesso em 2021 ago 12]; 19(3):267-78. doi: https://doi.org/10.1590/S0104-56872007000300005

30. World Health Organization. The World Health Report 2005: Make every mother and child count. World Health Organization, 2005. Disponível em: https://www.who.int/reproductivehealth/publications/maternal perinatal health/92 41562900/en/

RECEBIDO: 31/07/2021

ACEITO: $17 / 12 / 2021$ 